\title{
Polyethylene glycol versus vegetable oil based bisacodyl suppositories to initiate side-lying bowel care: A clinical trial in persons with spinal cord injury
}

\author{
Steven A Stiens ${ }^{1}$, William Luttrel ${ }^{2}$ and Joseph E Binard ${ }^{3}$ \\ ${ }^{1}$ VA Puget Sound Health Care System, Department of Rehabilitation Medicine, University of Washington, Seattle, \\ Washington; ${ }^{2}$ Veterans Administration Medical Center, Spinal Cord Injury Unit, Palo Alto, California; ${ }^{3}$ Veterans \\ Administration Medical Center, Departments of Urology \& Family Medicine, University of South Florida, Tampa, \\ Florida, USA
}

\begin{abstract}
Introduction: Neurogenic bowel dysfunction resulting from spinal cord injury (SCI) frequently requires bowel care (BC) with stimulant suppositories for initiation of effective defecation. The excessive time required for $\mathrm{BC}$ and bowel complications have limited quality of life after SCI.

Objective: To test the hypothesis that: the time required for bowel care with bisacodyl suppositories can be reduced by substituting a polyethylene glycol base (PGB) for the traditional hydrogenated vegetable oil base (HVB) in the suppository.

Setting: Inpatient SCI medicine unit.

Subjects: Fourteen persons with SCI with chronic stable paralysis from upper motor neuron SCI for greater than one year with a stable HVB bisacodyl suppository initiated BC.

Design: Crossover Controlled.

Method: Subjects received HVB bisacodyl suppositories for six sequential BC sessions and then were crossed over to PGB bisacodyl suppositories for six more BCs.

Outcome measures: $\mathrm{BC}$ event times were utilized to derive $\mathrm{BC}$ intervals: suppository insertion to first flatus = Time to flatus, first flatus until the beginning of stool flow = Flatus to stool flow, begin stool flow until end stool flow = Defecation period, end stool flow until end of clean up $=$ Clean up, and suppository insertion until end clean up $=$ Total bowel care time .

Results: The data included two groups of BC sessions: HVB $(n=84)$ and PGB $(n=81)$. Mean times in minutes and $P$ values from $t$ tests for paired samples yielded: Time to flatus: (HVB 31, PGB $12.8 P<0.002$ ), Defecation period: (HVB 58, PGB 32, P<0.0005), Clean up: (HVB 1.9, PGB 3.2 $P=0.165$ ), Total bowel care time: (HVB 102, PGB $51.2 P<0.0005$ ).

Conclusion: This analysis suggests that PGB based bisacodyl suppositories may stimulate reflex defecation sooner and shorten the Total BC Time as compared with HVB bisacodyl suppositories.
\end{abstract}

Keywords: colon; spinal cord injury; constipation; bisacodyl; incontinence; suppository

\section{Introduction}

Spinal cord injuries (SCI) result in upper or lower motor neuron damage that frequently produces neurogenic bowel dysfunction. Both the upper motor neuron (UMN) and lower motor neuron (LMN) patterns of bowel dysfunction are characterized by fecal retention and uniformly require a comprehensive plan of management (bowel program) that consists of diet, oral medications and a scheduled evacuation procedure (bowel care) to avoid impaction and incontinence. $^{1,2}$ Classically, bowel care has been a time consuming process that has been detrimental to

Correspondence: SA Stiens, MD, MS, Department of Rehabilitation Medicine RJ-30, University of Washington, BB938 Health Sciences Building, 1959 NE Pacific, Seattle, WA 98195, USA quality of life. ${ }^{3}$ Many people with SCI rank neurogenic bowel dysfunction as a major life limiting problem. ${ }^{4-7}$ Bowel care regimens can last longer than $3 \mathrm{~h}$ and still produce insufficient results. ${ }^{8}$ Consequently, in spite of tedious and exacting bowel care regimens, serious problems with bowel evacuation are still reported from as many as $20 \%$ of people with SCI. ${ }^{4,6}$

Suppositories containing an active laxative ingredient dispersed in a base substance, are frequently the mode of presentation for the stimulus of the chemical defection reflex. Bisacodyl is an active ingredient in many rectal chemical stimulant preparations for defecation. This compound, a diphenylmethane derivative (bis (p-acetoxyphenyl)-2-pyridylmethane) 
which was first introduced for use as a laxative in 1953 due to its structural similarity to phenolphthalein. Bisacodyl which acts as a contact laxative is practically insoluble in water and sparingly soluble in alcohol. Given rectally in a water suspension it is dispersed along the colonic mucosa. The colonic response can be detected with mucosal electromyography. Bisacodyl then acts within as little as 3 min to suppress rhythmic stationary spike wave activity and increase spasmodic propulsive peristaltic spike activity. ${ }^{9}$ Thereafter, reflex defecation ensues.

Bisacodyl can be administered in many preparations including suppositories, enemas, mini enemas, and solutions. ${ }^{10}$ For independent bowel care after SCI typically, $10 \mathrm{mg}$ suppositories are used due to ease of insertion with dexterity deficits and improved anal retention without voluntary external anal sphincter constriction. The most common suppository preparation includes bisacodyl powder dispersed within a hydrogenated vegetable oil base (HVB). ${ }^{10}$ Anecdotal reports suggest bisacodyl suppositories with a vegetable oil base often require a prolonged period to produce defecation and can cause continued mucosal irritation with resultant mucus accidents hours after bowel care is completed. As a result, water-miscible suppositories have been introduced. We have evaluated the effect of polyethylene glycol polymer based (PGB) bisacodyl suppositories using a single subject design. ${ }^{11} \mathrm{We}$ found that defecation was initiated more rapidly with a resultant significant shortening of the total bowel care time.

This study was carried out to compare the effectiveness of hydrogenated vegetable oil based and polyethylene glycol based bisacodyl suppositories in triggering and producing reflex defecation in a cohort of SCI subjects during bowel care administered within a hospital setting.

\section{Methods}

Recruitment focused on inpatient SCI persons at the Tampa Veterans Affairs Medical Center SCI unit. Subjects were invited to enter the evaluation if they had been SCI for greater than 1 year, had an upper motor neuron lesion, and used HVB bisacodyl suppositories in a regular, stable bowel care regimen. Subjects were excluded if there was a recent history of constipation, diarrhoea, or medication that could adversely affect bowel function. Baseline demographic information on each subject included: age, years since SCI, SCI level, bulbocavernous reflex, anocutaneous reflex, anal tone, and phasic reflexes of the lower extremities. $^{1}$

The two types of bisacodyl suppositories used in the study differed only in the base used for dispersion of the active ingredient. The HVB suppositories utilized contained $10 \mathrm{mg}$ bisacodyl USP in a hydrogenated vegetable oil base (UDL Lab, Rockford, USA). The PGB suppositories (Concepts in
Confidence, Islandia, NY, USA) contained $10 \mathrm{mg}$ bisacodyl dissolved in a mixed polyethylene glycol polymer base of two molecular weights: E1450 and E400.

Subjects received one HVB suppository for six sequential regularly scheduled bowel care procedures followed by one PGB per rectum for the following six $\mathrm{BC}$ sessions. Each bowel care procedure was performed by a staff nurse with the consistent technique and data collection. ${ }^{1}$ All subjects were positioned side-lying with the left side down and knees flexed. At the beginning of each bowel care session, either a PGB or a HVB bisacodyl suppository was inserted and positioned against the mucosal surface of the rectum at a finger length from the anus.

The presence or absence of stool in the rectal vault was recorded. The time of insertion was considered time zero and the progress of bowel care was documented with time parameters (Figure 1). ${ }^{11}$

Bowel care events were used to separate the total bowel care time period into discrete intervals recorded in minutes: First flatus (ends the interval from suppository insertion until the first gas is passed), Begin stool flow (marks the beginning of the defecation interval), End stool flow (marks the end of defecation interval), and Wait until transfer (marks the end of the period of waiting after stool flow has ended, the subjective end of the bowel care). ${ }^{1,11}$ For the purpose of this study bowel care was deemed complete at the end of the perianal clean up (End clean up) after waiting to see that no further stool was expelled. Our subjects had bowel care in bed and did not

\section{Events and Intervals of Bowel Care}

Intervals:

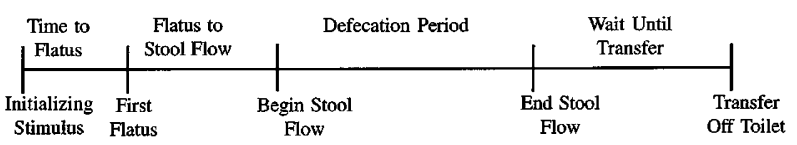

Events:

Figure 1 Bowel care session events separate the total bowel care period into four discrete intervals. Bowel care begins with suppository insertion. First flatus ends the first interval Time to flatus (suppository insertion until first gas is passed). Begin stool flow ends the second interval termed Flatus to stool flow and begins the Defecation period. End stool flow represents the time when Defecation period has immediately ceased. The time of Transfer off the toilet ends the Wait until transfer period which represents the time spent to insure that the bowel care is over. The time of Transfer off the toilet ends bowel care. If there is no transfer then the end of bowel care is defined as the end of clean up of the perianal area End clean up. The total bowel care time is the period from the suppository insertion until the Transfer off the toilet or end clean up 
consistently require a transfer at the end of the procedure.

Digital stimulations were carried out in a circular motion with the gloved lubricated index finger in an attempt to dilate the external and internal anal sphincters and the distal rectum stimulating reflex peristalsis. ${ }^{1}$ Digital stimulations were done if stool flow stopped or slowed during the evacuation process (approximately one every $10 \mathrm{~min}$ ). Digital examination of the rectal vault followed the end of stool flow to assure complete evacuation. The number of digital stimulations required for each bowel care session was recorded.

The amount of stool produced with each bowel care session was recorded using the following ordinal scale based on a comparison to the subject's previous bowel care results before the study: (0) no stool, expelled; (1) small-less than expected; (2) moderatestool volume as expected or (3) large-larger than expected results. The time, amount and frequency of mucus and stool accidents were recorded if they occurred between bowel care sessions. Bowel incontinence was defined as each individual episode of unplanned stool evacuation that occurred between the end of clean up marking the end of bowel care and the insertion of the suppository for the next bowel care regimen.

\section{Statistical analysis}

Mean interval durations for the collective trials with each suppository for each subject were calculated and analysed with $t$-tests for paired samples using the Statistics Program for the Social Sciences for Windows (SPSS for Windows).

\section{Results}

\section{Subject demographics}

The 14 subjects were all male and included four incomplete and ten with complete spinal cord injuries. The average age was 53.4 years and the average years since SCI was 18.3. SCI levels ranged from C3 to L1. The bulbocavernosis reflex was present in $14 / 14$ and the anal cutaneous reflex was present in 9/14. Anal tone was high in $2 / 14$, normal in $3 / 14$ and reduced in $9 /$ 14.

\section{Bowel care intervals}

The means and $P$ values were as follows: Time to flatus (Suppository insertion until first gas expulsion) $\mathrm{HVB}=31 \mathrm{~min}, \mathrm{PGB}=12 \mathrm{~min} \quad P<0.002$. Defecation period (period of stool flow) $\mathrm{HVB}=58 \mathrm{~min}$, $\mathrm{PGB}=32$ min $P<0.0005$, Clean up (end of stool flow to end of clean up $=\mathrm{HVB}=1.9 \mathrm{~min}, \mathrm{PGB}=3.2 \mathrm{~min}$ $P=0.165$ (Figure 2). The means of the Total bowel care time (suppository insertion until the end of clean up) was $\mathrm{HVB}=102 \mathrm{~min}$ and $\mathrm{PGB}=51.2 \mathrm{~min}, P<0.0005$, a statistically significant difference. Means of the Total bowel care time for individual subjects were consistently lower with the PGB suppository, (Figure 3).

\section{Digital stimulations and stool results}

The numbers of digital stimulations required for the bowel care (BC) sessions were averaged (HVB 5.0, PGB 3.2) and compared using $t$-tests for paired samples $P<0.0005$. The amount of stool produced by the $\mathrm{BC}$ was compared for HVB and PGB, and revealed no significant difference $P=0.3505$. Sixteen stool incontinence episodes were recorded between $\mathrm{BC}$ sessions: 15 after HVB and 1 after PGB.

\section{Discussion}

This open label clinical trial investigated the statistical and clinical significance of the use of polyethylene glycol based (PGB) bisacodyl suppositories versus the use of vegetable oil based (HVB) bisacodyl suppositories using a sequential presentation of each agent. We found comparable bowel care effectiveness for the amount of stool eliminated with a reduction in bowel care time by approximately half using the PGB. These time differences were observed with similar volumes of stool results for bowel care sessions. This improvement in efficiency demonstrates a statistically and clinically significant result for these subjects.

The mean bowel care interval that was most reduced was Time to flatus. We have previously

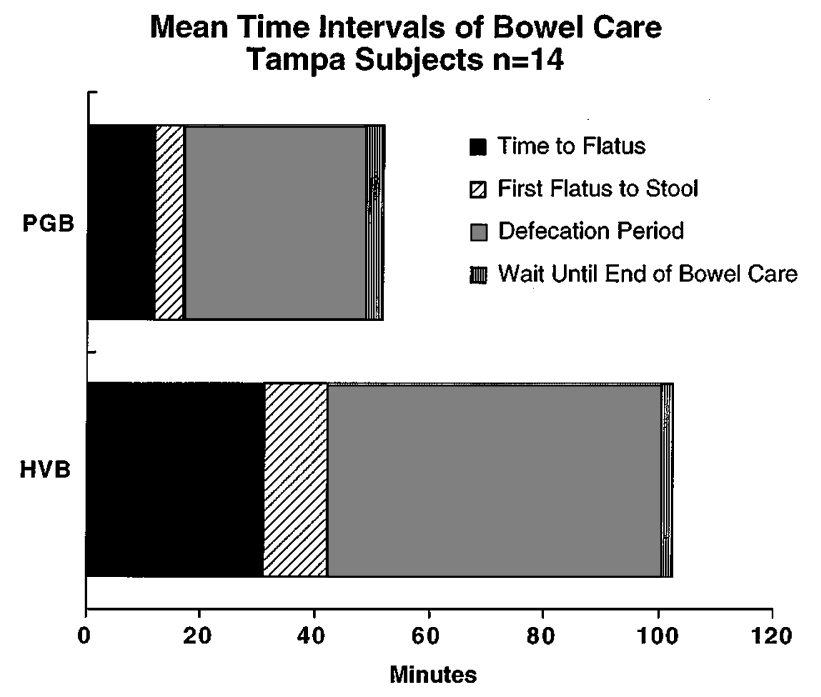

Figure 2 The time intervals of the bowel care periods from all 14 subjects studied were averaged and presented in minutes for PBG (polyethylene glycol based) and HVB (hydrogenated vegetable oil based) bisacodyl suppositories. The Time to flatus was significantly longer after HVB suppository insertion. The Flatus to stool flow period showed no significant difference between the agents. The Defecation period was significantly shorter with use of the PGB suppository 


\section{Total Bowel Care Time}

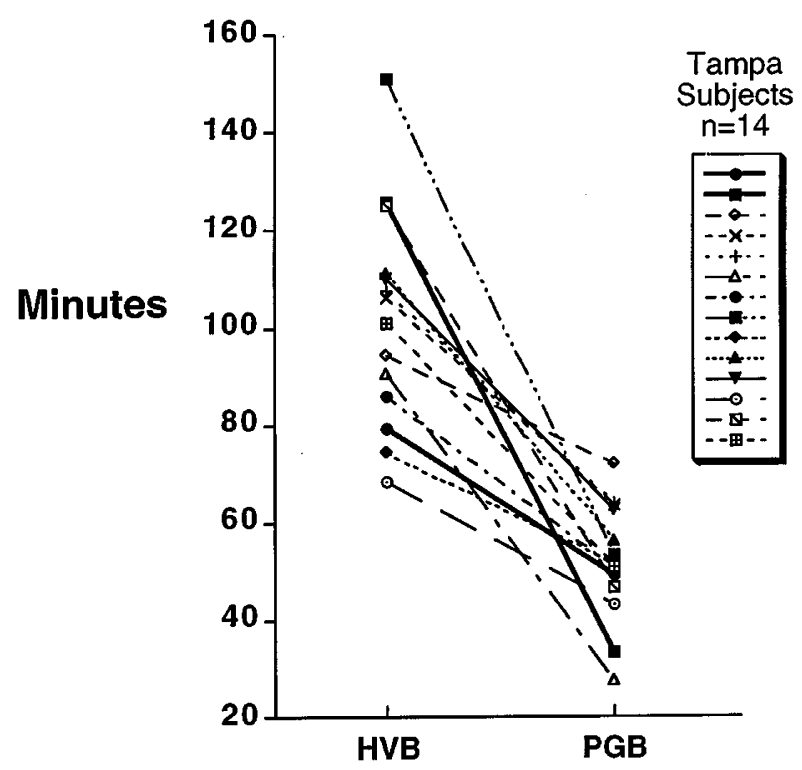

Figure 3 This scatter plot represents mean times in minutes for the Total bowel care periods after administration of PGB and HVB suppositories for each subject. The lines connecting the means illustrate the reduction in time required for pharmacologically triggered defecation

suggested that this time difference may be due to the rate and amount of bioavailability of the active ingredient (bisacodyl) in the suppositories. ${ }^{11}$ Parrott also found polyethylene glycol to be a superior suppository base that facilitated rapid absorption of salicylate. $^{12}$ When polyethylene glycol liquifies in response to body heat it may dissolve and disperse bisacodyl more effectively on mucous membranes.

We also observed a reduction in the time of the defecation or stool flow period with PGB. This may be due to a more widespread dispersion of the PGB base with bisacodyl producing a stronger and more sustained reflex stool propulsion. We conclude that the simple substitution of a PGB bisacodyl suppository for a HVB one can reduce bowel care time by almost one half. In our study group this saved up to $1 \mathrm{~h}$ per $\mathrm{BC}$ session for the average patient, improving quality of life.

Bowel care with HVB included more digital stimulations than with PGB. Our protocol required digital stimulations approximately every $10 \mathrm{~min}$ to optimize efficiency of evacuation. In spite of chemical and mechanical stimulation the average defecation period of the HVB initiated bowel care $(58 \mathrm{~min})$ was significantly longer than with PGB (32 min). As a result, more digital stimulations were required during the defecation period with HVB. Although some persons utilize chemically stimulated bowel care routines that do not include digital stimulation, we postulate that chemical and mechanical stimulation complement one another in promoting efficient stool elimination. Our study was designed with the manipulation of only one variable (bisacodyl suppository) and therefore cannot fully evaluate the effectiveness of digital stimulation.

The frequency of unplanned stool evacuations between bowel care sessions was much higher following $\mathrm{BC}$ with $\mathrm{HVB}$ bisacodyl suppositories. The hydrophobic vegetable oil base (HVB) may sequester the bisacodyl and gradually release it to diffuse onto the colon mucosae. This may produce a prolonged stimulus to defecation and an irritation that produces excess mucus secretion in some subjects. The clinician should sensitively inquire about involuntary evacuations experienced by patients and be aware that rectal stimulants may be associated with these events. Alternatives for redesign of the bowel care regimen to improve continence could include use of half of a suppository, consideration of other rectal stimulants (PGB bisacodyl suppositories, therevac mini enemas, glycerine suppositories) or transition to bowel care initiated and sustained by repeated digital rectal stimulation. ${ }^{1}$ Reduction in total bowel care time and frequency of incontinence should be foci of intervention in the establishment and refinement of the bowel program as these parameters have been significantly associated with emotional upset in coping with neurogenic bowel after SCI. ${ }^{4}$ The management of neurogenic bowel dysfunction has been recently reviewed in an evidence based clinical guideline. ${ }^{13}$

\section{Acknowledgements}

We are very grateful to the nursing staff and patients of the James A. Haley Veterans Hospital, Spinal Cord Injury Unit for their support of research. The authors would like to thank Tammy Pidde RN for her enthusiasm and commitment to education of patients and staff in the most effective bowel care practices. We appreciate the continued efforts of the collaborators in the Bowel Care Video Education Project at the Puget Sound Health Care System, Seattle Division Hospital: Kathy Chadband, Marguerite David MSW, Tammy Piddie RN and Brenda Veland. This project was funded in part by a grant from the Paralyzed Veterans of America, Education and Training Foundation, Video Education in Assisted Bowel Care for Persons with SCI: Intervention, Video Production and Effectiveness Assessment, \#316-96. Laxative suppositories (PGB) bisacodyl $10 \mathrm{mg}$ NDC 57648-002-01, were supplied by Concepts in Confidence 1797-32 Veteran's Hwy, Islandia, NY, 11722, USA 1-800-822-4050, and laxative suppositories (HVB) bisacodyl $10 \mathrm{mg}$ were supplied by UDL Lab Rockford, Ill, 61103, USA, 1-800-435-5272. This paper was presented at the Annual Assembly of the American Paraplegia Society, Las Vegas, Nevada, September 1995.

\section{References}

1 Stiens S, Bierner-Bergman S, Goetz L. Neurogenic bowel dysfunction after spinal cord injury: Clinical evaluation and rehabilitative management. Arch Phys Med Rehabil 1997; 78: S86-S102. 
2 Stiens S, Goetz L. Neurogenic bowel dysfunction. In: O'Young B, Young M, Stiens S, (eds). Physical Medicine and Rehabilitation Secrets. Hanley \& Belfus, 1997; pp 460-464.

3 Banwell J, Creasey G, Aggarwal A, Mortimer J. Management of the neurogenic bowel in patients with spinal cord injury. Urologic Clinics of North America 1993; 20: 517-526.

4 Glickman S, Kamm MA. Bowel dysfunction in spinal cord injury patients. Lancet 1996; 347: $1651-1653$.

5 Levi R, Hulting C, Nash M, Seger A. The Stockholm spinal cord injury study: 1. Medical problems in a regional SCI population. Paraplegia 1995; 33: 308-315.

6 Stone J, Nino-Murcia M, Wolf V, Perkash I. Chronic gastrointestinal problems in spinal cord injury patients: a prospective analysis. Am J Gastroenterology 1990; 85: 114-119.

7 White M, Rintala D, Hart K, Furher M. Sexual activities, concerns and interests of women with spinal cord injury living in the community. Am J Phys Med Rehab 1993; 72: 372-378.

8 Frost F. Gastrointestinal dysfunction in spinal cord injury: Disorders of the bowel and bowel emptying. In: Green D, (ed). Medical Complications of Disability. Rockville, MD: Aspen Publications, 1990
9 Schang J, Hemond M, Hebert M, Pilote M. Changes in colonic myoelectric spiking activity during stimulation by bisacodyl. Can J Physiol Pharmacol 1986; 64: 39-43.

10 Barnhart E. Physicians' Desk Reference for Nonprescription Drugs. Oradell, NJ: Medical Economics Company, 1990.

11 Stiens SA. Reduction in bowel program duration with polyethylene glycol based bisacodyl suppositories. Arch Phys Med Rehabil 1995; 76: 674-677.

12 Parrott E. Salicylate absorption from rectal suppositories. Journal of Pharmaceutical Sciences 1971; 60: 867-872.

13 King RB, Biddle AK, Braunschweig C, Chen D, Cowell F, Dingur MC, Hammond MC, Hartley C, Longo WE, MathewsKirk P, Nelson A, Steins SA. Neurogenic bowel management in adults with spinal cord injury, Consortium for Spinal Cord Medicine, Paralyzed Veterans of America. 1998; $39 \mathrm{p}$ 